Apotheek Meindersma, Zwolseweg 2, 74I2 AN Deventer, Mevr. Drs. L. M. THOMAN-VosKuYL. Apotheek Dordrecht Zuid, Sandenburg Ia, 3328 EA Dordrecht, Drs. W. H. LIM.

Apotheek Heggen, Akerstraat Noord 43, 643 I HL Họensbroek-Treẹbeek, Drs. F. H. TH. HOOGENBERK.

Apotheek Tot hulp der menschheid, Rosmolen I3, 23 I7 PL Leiden, Drs. D. DE ROODE. Apotheek Boehmer, Florijn I9-2I, 2353 TC Leiderdorp, Drs. H. HUIZER.

Apotheek Monnickendam, Pierebaan I, I I4I GV Monnickendam, Drs. H. H. VAN ROOIJ. Apotheek de Groenberg BV, Molenstraat 29, 5688 AC Oirschot, Drs. P. B. MERTENS.

Apotheek \& Lab. van Kempen, Voorstad IO, 6I3 I CR Sittard, Dr. H. G. J. VAN KEMPEN. Apotheek van den Berg BV, Vleesstraat 24, 400I BC Tiel, Drs. A. H. VAN DEN BERG.

Apotheek Dorestede, Karel de Grotestraat 55, 3962 cK Wijk bij Duurstede, Mevr. Drs. M. W. v. D. WERKEN-KWAKKEL.

Apotheek Messchaert, Dennenlaan I3I, I I6I CN Zwanenburg, Mevr. Drs. J. M. J. DE BooY.

\title{
BEËINDIGING BEROEPSUITOEFENING
}

Drs. H. L. J. BECH, Beethovenlaan I23, 255I KC 's-Gravenhage.

Drs. M. A. ROBLES, Hilverbeekstraat IO, I024 KL Amsterdam.

Mej. Drs. A. RÖMER, Oostplein I I3, 30I I KW Rotterdam.

\section{LEDENLIJST}

AANMELDING A ALS LID

Belanghebbenden wordt gewezen op de artikelen 3 en 5 van het Huishoudelijk Reglement.

Mevr. W. M. BöTTGER, Warmoesstraat 54, 9724 JM Groningen, (dept. Groningen).

H. BRACHT, Jonkerlaan I5, 2242 GA Wassenaar, (dept. 's-Gravenhage).

R. DENEE, p/a Donkerstraat 50, Postbus 148, 3840 AC Harderwijk.

K. E. HAGENAAR, Cruquiuslaan 157, 2332 EA Leiden, (dept. 's-Gravenhage).

A. B. W. G. VAN OPPENRAAY, Baalsestraat 5, Bemmel, (dept. Gelderland).

H. TAlSma, Pelikaanhof I02, 2312 EE Leiden, (dept. Leiden).

AANGENOMEN ALS LID

Mevr. J. M. J. DE BOOY, t. Apotheek Messchaert, Dennenlaan 131, I I61 CN Zwanenburg, (02907) 5777. Privé: Morsweg 56d, 2312 AE Leiden, (07I) 131208, (dept. Amsterdam).

WIJZIGINGEN VAN NAMEN, ADRESSEN EN TEleFOONNUMMERS

H. M. VAN AKKERVEEKEN, $f$. Arembergstraat 18, 5583 CD Waalre, (04904) 6127.

Mevr. J. M. ASCHERMAN-v. D. BURG, $f$. Schoutenstraat 40, 6826 MB Arnhem, (085) 635227.

J. A. M. H. BACKBIER, g. Apotheek \& Lab. van Kempen, Voorstad I0, 6I3 I CR Sittard, (04490) I 2796.

H. L. J. ВеCH, $f$. Beethovenlaan I23, 255 I KC 's-Gravenhage. (070) 682516.
A. H. VAN DEN BERG, $t$. Apotheek van den Berg BV, Vleesstraat 24, 4001 BC Tiel, (03440) 3802 en 3953.

Mevr. y. H. VAN BEUSEKOM-BOomSMA, $f$. Beresteynstraat 2, 225I KC Voorschoten, (017I7) 2520.

J. P. Boelstra, g. Privé: Amalia van Solmslaan 14, 9602 GM Hoogezand, (05980) 22677.

Mevr. M. A. BOONACKER, $t$. Voorhof Apotheek, Papsouwselaan 230, $2624 \mathrm{EH}$ Delft, (O15) 612772, (thans dept. 's-Gravenhage).

Mevr. A. c. C. Bos, g. Apotheek den Heyer, Keizerstraat I67, 2584 BE 's-Gravenhage, (070) 5404I2.

Mevr. M. BRasz, $t$. Privé: van Eerbeeklaan I2, I422 GG Uithoorn, (02975) 62147.

W. BREDENOORD, g. Apotheek Robles, Waterlandplein 20, I024 LV Amsterdam, (020) 363467.

Mevr. c. E. BRIENEN, g. Apotheek Dorestede, Karel de Grotestraat 55, $3962 \mathrm{CK}$ Wijk bij Duurstede, (03435) 3603.

J. W. BRIENEN, g. Apotheek van den Berg BV, Vleesstraat 24, 400I BC Tiel, (03440) 3802 en 3953, (thans dept. Gelderland).

J. BRON, $i$. Privé: van Breestraat 170", I071 zX Amsterdam, (020) 643439.

Mevr. C. L. BUIJS-TJOE AWIE, $g$. Sumatra Apotheek, Sumatrastraat 64a, I094 NG Amsterdam, (020) 650905.

J. G. VAN DAM, $f$. Tinnegieter 75, I625 AT Hoorn, (02290) 3 I 867 .

W. DERMER, g. Privé: Gaailaan 3, 3722 ZH Bilthoven, (030) 790295 .

J. J. DONKERS, g. Apotheek Peterse, Kromstraat I08, 5345 AE Oss, (04I 20) 25339.

Mevr. J. Е. M. TEN DIJK, $f$. Plantijnstraat 38 , flat 38,2321 JD Leiden, (O7I) 762326. 